\title{
CONTAMINACIÓN POR CADMIO EN ALIMENTOS MARINOS, LIMA - 2015
}

\author{
Cadmium pollution in seafood, Lima - 2015 \\ Gloria M. Marín, Mesías M. García \\ Facultad de Farmacia y Bioquímica, Universidad Nacional Mayor de San Marcos
}

\section{RESUMEN}

En la presente investigación se determinaron las concentraciones de cadmio en alimentos marinos, y se compararon con los contenidos máximos según el Reglamento (CE) № 1881/2006 y modificatoria Reglamento (UE) № 488/2014 de la Comisión de la Unión Europea. Las muestras fueron recolectadas del Terminal Pesquero de Villa María del Triunfo. Los análisis se realizaron por espectrofotometría de absorción atómica. Los resultados fueron: en pescados (jurel: Trachurus picturatus murphyi) el promedio de las concentraciones de cadmio fue $0,35 \mathrm{mg} / \mathrm{kg}$ peso fresco, muy por encima del valor permitido que es o,o5 mg/kg peso fresco. En crustáceos (langostinos: Litopenaeus vannamei) el promedio fue o,42 $\mathrm{mg} / \mathrm{kg}$ peso fresco, siendo inferior al límite máximo permitido que es $0,50 \mathrm{mg} / \mathrm{kg}$ de peso fresco. El límite máximo en moluscos bivalvos es 1,oo $\mathrm{mg} / \mathrm{kg}$ peso fresco y se encontraron concentraciones diferentes en especies distintas, así las conchas blancas (Semele sp) y conchas de abanico (Argopecten purpuratus) no superan los límites permitidos; los choros (Aulacomya atra) están en el límite; las machas (Mesodesma donacium) y almejas (Gari solida) superan los valores límites. Por último, en moluscos cefalópodos (pota: Dosidicus gigas) el promedio fue 1,11 mg/kg peso fresco, siendo ligeramente mayor al límite permitido que es 1,oo mg/kg peso fresco.

Palabras clave: Cadmio, alimentos marinos, contaminación.

\section{SUMMARY}

In the present investigation, cadmium concentrations in seafood were determined and compared with the maximum levels of Commission of the European Union in its Regulation (EC) № 1881/2006 and amending Regulation (EU) No 488/2014. Samples were collected from the fish market of Villa Maria del Triunfo. Analyses were performed by atomic absorption spectrophotometry. The results were: in fish (horse mackerel: Trachurus picturatus murphyi) the average cadmium concentrations was $0,35 \mathrm{mg} / \mathrm{kg}$ fresh weight, well above the allowable value which is $0,05 \mathrm{mg} / \mathrm{kg}$ fresh weight. In crustaceans (prawns: Litopenaeus vannamei) the average was $0,42 \mathrm{mg} / \mathrm{kg}$ fresh weight, being below the maximum allowed limit which is $0,50 \mathrm{mg} / \mathrm{kg}$ fresh weight. The ceiling in bivalve molluscs is $1,00 \mathrm{mg} / \mathrm{kg}$ fresh weight and different concentrations were found in different species and in white shells (Semele sp) and scallops (Argopecten purpuratus) do not exceed the permitted limits; the mussels (Aulacomya atra) are borderline; the machas (Mesodesma donacium) and clams (Gari solida) exceed the limit values. Finally, cephalopods (giant squid: Dosidicus gigas) the average was $1,11 \mathrm{mg} / \mathrm{kg}$ fresh weight, being slightly higher than the allowable limit 1,oo mg / kg fresh weight.

Keywords: Cadmium, seafood, pollution.

\section{INTRODUCCIÓN}

$\mathrm{E}$ 1 cadmio es un xenobiótico y, por tanto, un metal tóxico y no esencial para el organismo que se acumula en los tejidos, se absorbe por las vías respiratoria y digestiva. Las intoxicaciones por cadmio resultan de la exposición ambiental e industrial, encontrándose en algunos alimentos (especialmente bivalvos, en concentraciones del orden de $1 \mathrm{mg} / \mathrm{Kg}$ ), en el humo del tabaco y en el aire atmosférico de las aglomeraciones urbanas ${ }^{(1)}$. Es es tóxico para todos los sistemas y funciones humanas o animales, tendiendo a ser almacenado en hígado, riñón y pulmones e inhibiendo a las enzimas con grupos sulfhidrilo en el sitio activo ${ }^{(2)}$.
Existen investigaciones sobre el cadmio, de las cuales dos se hicieron en el Perú. Juárez ${ }^{(3)}$ realizó una investigación de metales pesados y su efecto en la agricultura, en la cual determinó que los niveles de concentración anuales para cadmio fueron menores a $0,05 \mathrm{mg} / \mathrm{L}$ (aceptable para el regadío de hortalizas) para los ocho años evaluados (1997-2004), no existe impacto de contaminación por cadmio en ninguna parte de la cuenca del río Rímac. Ibárcena ${ }^{(4)}$ elaboró un estudio de contaminación por metales pesados, y encontró que el nivel promedio de cadmio en agua de mar era de $0,53 \mu \mathrm{g} / \mathrm{L}$ y no representaba un riesgo para la salud; mientras que en sedimentos marinos el promedio de cadmio encontrado fue de $0,16 \mu \mathrm{g} / \mathrm{L}$ que es un valor por debajo de los límites permisibles, esto 
puede deberse a la baja cantidad de materia orgánica en las zonas de muestreo donde predominaba la arenilla.

A nivel latinoamericano Pérez et al. ${ }^{(5)}$ realizaron en Argentina un estudio sobre el contenido de plomo, cromo y cadmio en moluscos comestibles, entre ellos Aulacomya atra (cholga) y Mytilus edulis platensis (mejillón), que son recolectados como recurso alimentario. Se encontró que los niveles más altos (en peso seco) de cadmio fueron de 5,72 $\mu \mathrm{g} / \mathrm{g}$ en mejillones de la zona Punta Maqueda, lugar alejado de la actividad antropogénica. Los valores de cadmio y plomo hallados no superan los límites máximos establecidos por la Secretaría Nacional de Sanidad y Calidad Agroalimentaria, sin embargo se han observado valores cercanos al ISTP (ingesta semanal tolerable provisional) para el cadmio. FríasEspericueta et al. ${ }^{(6)}$ publicaron un estudio, en México, sobre cadmio y plomo en organismos de importancia comercial encontrándose concentraciones de ambos relativamente altas, por lo cual es posible concluir que, aunque sin todavía alcanzar niveles críticos, existe un problema ambiental en esta zona del país.

Estudios en Europa (7), revelan altas concentraciones de cadmio en ostiones y mejillones caballos cosechados en aguas noruegas, con niveles por encima de los límites máximos de la Unión Europea (UE). Se analizaron la glándula digestiva del ostión y el riñón del mejillón, encontrándose que el contenido de cadmio (3,4-8,4 mg/kg) excede los límites máximos de la UE si se mide todo el contenido del molusco, pero la mayor parte se encuentra en la glándula digestiva. España puso de manifiesto que los pescados y mariscos son los alimentos que contribuyen de forma más significativa a la ingesta de contaminantes a través de la dieta; con respecto al cadmio los niveles más elevados se detectaron en la almeja y el mejillón, con o,143 $\mu \mathrm{g} / \mathrm{g}$ de peso en fresco y $0,132 \mu \mathrm{g} / \mathrm{g}$ de peso en fresco, respectivamente. Las concentraciones de cadmio que presentan las especies estudiadas en este país se encuentran por debajo de los valores establecidos por la Comunidad Europea (CE) ${ }^{(8)}$.

Fue objetivo de la presente investigación determinar y contrastar la concentración de cadmio en alimentos marinos con los límites permisibles.

\section{MATERIALES Y MÉTODOS}

Muestreo: los ejemplares de pescado jurel (Trachurus picturatus murphyi), langostinos (Litopenaeus vannamei), conchas abanico (Argopecten purpuratus), conchas blancas (Semelesp), choros (Aulacomya atra), almejas (Gari solida), machas (Mesodesma donacium) y pota (Dosidicus gigas) fueron colectados al azar del Terminal Pesquero de Villa María del Triunfo, provenientes del litoral de la región Lima, sub área 3: Chorrillos - Islas Pachacámac.

Tratamiento de las muestras: se pesaron aproximadamente 100 gramos de cada una de las muestras, en tres oportunidades diferentes, y fueron envasadas en frascos de plástico de polietileno, rotuladas, conservadas a $4^{\circ} \mathrm{C}$ y trasladadas inmediatamente a la Unidad de Servicios de Análisis Químicos (USAQ) de la Facultad de Química e Ingeniería Química de la Universidad Nacional Mayor de San Marcos para determinar la concentración de cadmio por el método de espectrofotometría de absorción atómica.

\section{Análisis por espectrofotometría deabsorción atómica}

Fundamento: la muestra solubilizada es aspirada y atomizada dentro de la llama de un espectrofotómetro de absorción atómica, equipado con lámpara de cadmio, y se mide a una longitud de onda de $228,8 \mathrm{gm}^{(9)}$.

Preparación de la muestra: se realizó digestión húmeda pesando 2,5 gramos de cada una de las muestras, por separado, en un beaker de $600 \mathrm{~mL}$. Se añadieron $25 \mathrm{~mL}$ deácido nítrico concentrado, secubrió con un vidrio de reloj, y se dejó hervir durante 30-45

Tabla 1. Promedios de las concentraciones de cadmio en alimentos marinos.

\begin{tabular}{llc}
\multicolumn{1}{c}{ Grupo } & \multicolumn{1}{c}{ Especie } & $\begin{array}{c}\text { Promedio: } \\
\text { mg/Kg peso fresco }\end{array}$ \\
\hline Pescados & Pescado (jurel: Trachurus picturatus murphyl) & 0,33 \\
Crustáceos & Langostinos (Litopenaeus vannamei) & 0,42 \\
& Conchas blancas (Semele sp) & 0,82 \\
& Conchas de abanico (Argopecten purpuratus) & 0,83 \\
Moluscos bivalvos & Choros (Aulacomya atra) & 1,00 \\
& Machas (Mesodesma donacium) & 1,28 \\
Moluscos cefalópodos & Almejas (Gari solida) & 1,39 \\
Fuente: propia. & Pota (Dosidicus gigas) & 1,11 \\
\hline
\end{tabular}


minutos para oxidar todo el material. Posteriormente se enfrió la solución y se agregó lentamente $10 \mathrm{~mL}$ de ácido perclórico al 70\%. Nuevamente se hizo hervir hasta que la solución quedó casi incolora. Se enfrió, filtró y diluyó hasta $100 \mathrm{~mL}$ con agua desionizada.

Procedimiento: Se homogenizó la muestra anterior en agitador rotatorio. Se realizó una disolución con Tritón $\mathrm{X}-100$ al $0,1 \%$ y ácido nítrico al 0,1\% (1:10). Luego se depositaron en una celda $540 \mu \mathrm{L}$ de la disolución y $60 \mu \mathrm{L}$ de la muestra problema. Se tomaron $15 \mu \mathrm{L}$ de la muestra

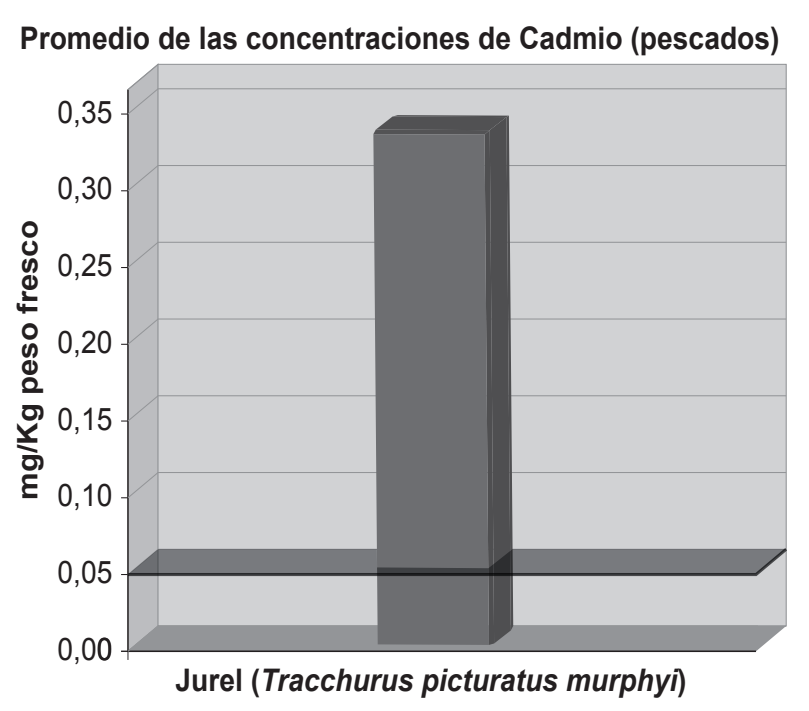

Figura 1. Promedio de las concentraciones de cadmio en pescados en $\mathrm{mg} / \mathrm{kg}$ de peso; la línea diferenciada es el contenido máximo permitido.

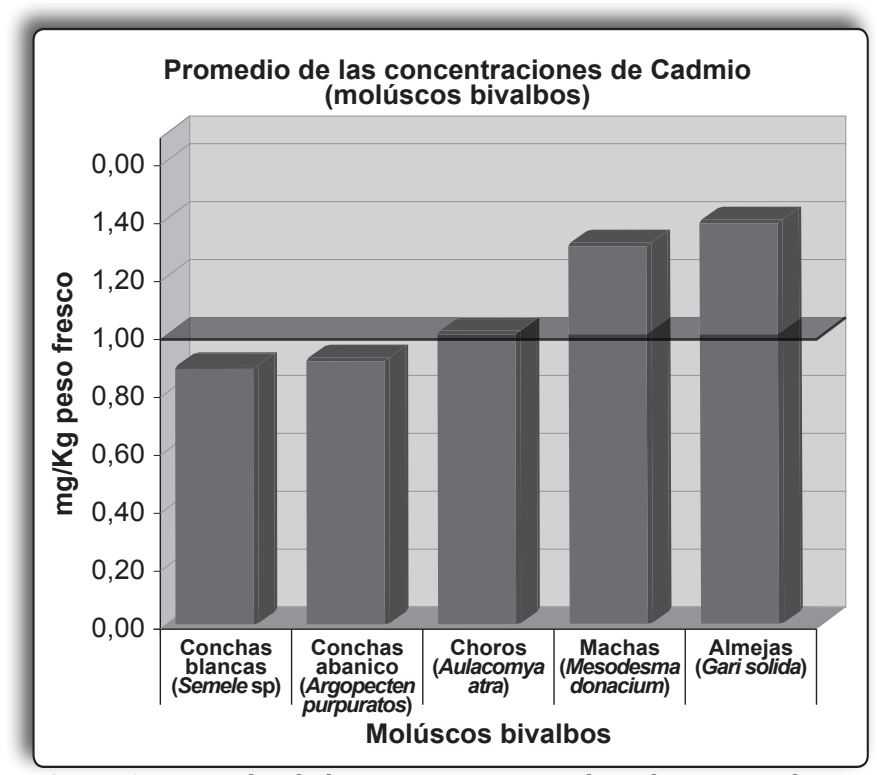

Figura 3. Promedio de las concentraciones de cadmio en moluscos bivalvos en $\mathrm{mg} / \mathrm{kg}$ de peso fresco; la línea diferenciada es el contenido máximo permitido. anterior y se agregaron $5 \mu \mathrm{L}$ de fosfato diácido de amonio. Finalmente se llevóa espectrofotómetro y se leyó 228,8 ym.

\section{RESULTADOS}

Los valores obtenidos de las muestras se compararon con los límites permitidos, representados por una línea diferenciada en cada figura.

Dichas comparaciones muestran que el promedio de las concentraciones de cadmio en pescados supera

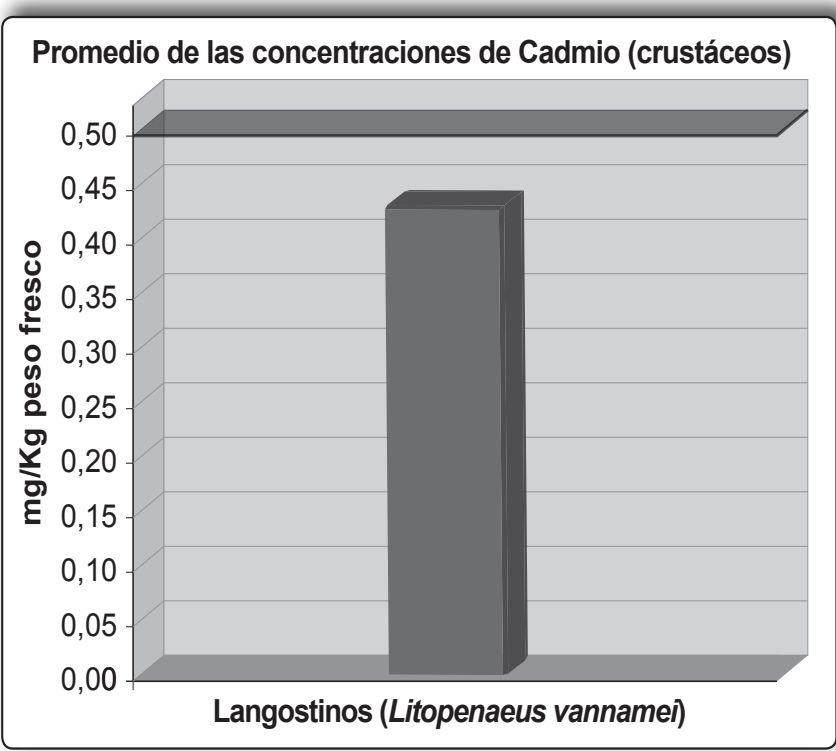

Figura 2. Promedio de las concentraciones de cadmio en crustáceos en $\mathrm{mg} / \mathrm{kg}$ de peso fresco; la línea diferenciada es el contenido máximo permitido.

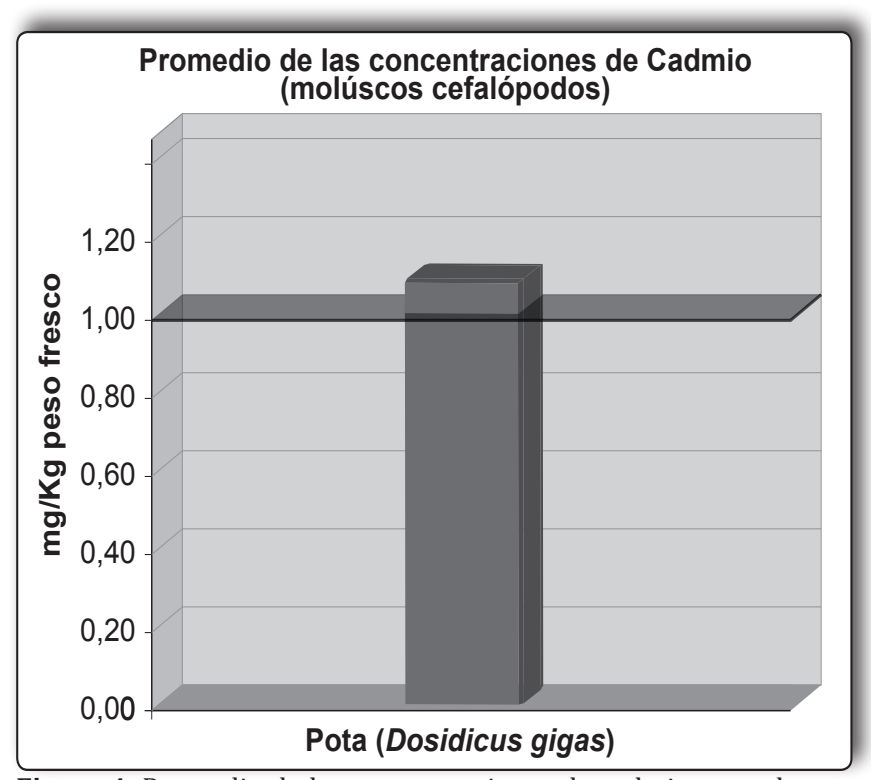

Figura 4. Promedio de las concentraciones de cadmio en moluscos cefalópodos $\mathrm{mg} / \mathrm{kg}$ de peso fresco; la línea diferenciada es el contenido máximo permitido. 
los límites permitidos (figura 1); en crustáceos langostinos- no los supera (figura 2); en cuanto a las cinco especies de bivalvos, dos no los sobrepasan, una se encuentra en el límite y dos están por encima (figura 3); mientras que en molúscos cefalópodos se superan ligeramente los límites (figura 4).

\section{DISCUSIÓN}

La Unión Europea ha establecido por norma el contenido máximo de determinados contaminantes en los productos alimenticios, en los que se incluyen pescados, moluscos cefalópodos, moluscos bivalvos y crustáceos ${ }^{(10)}$. Esta norma tuvo dos modificaciones ${ }^{(11,12)}$ que reducen el contenido máximo de cadmio en algunas especies de pescados de $0,10 \mathrm{mg} / \mathrm{kg}$ a $0,05 \mathrm{mg} /$ $\mathrm{kg}$ de peso fresco.

En pescados, la concentración promedio de cadmio en Trachurus picturatus murphyi fue $0,35 \mathrm{mg} /$ $\mathrm{kg}$ peso fresco, valor por encima de los $0,05 \mathrm{mg} / \mathrm{kg}$ peso fresco permitidos.

En lo referente a crustáceos, los niveles de cadmio en langostinos (Litopenaeus vannamei) fueron o,42 $\mathrm{mg} / \mathrm{kg}$ peso fresco, inferiores al límite máximo permitido de $0,50 \mathrm{mg} / \mathrm{kg}$ peso fresco.

El primer caso marca diferencia de lo reportado en Cataluña, donde los niveles no rebasan los límites establecidos, mientras que el segundo concuerda ${ }^{(8)}$.

Para moluscos bivalvos se tomaron cinco especies, pues el cadmio se almacena en mayores concentraciones en estos organismos. El límite máximo es de $1,0 \mathrm{mg} / \mathrm{kg}$ peso fresco y se encontraron concentraciones diferentes en especies distintas, así, en conchas blancas (Semele sp) y conchas abanico (Argopecten purpuratus) fueron 0,82 y $0,83 \mathrm{mg} / \mathrm{kg}$ peso fresco, respectivamente, valores que no superan los límites permitidos; los choros (Aulacomya atra) están en el límite con 1,oo $\mathrm{mg} / \mathrm{kg}$ peso fresco; las machas (Mesodesma donacium) y almejas (Gari solida) con 1,28 y 1,39 mg/kg peso fresco, respectivamente, sí superaron los valores límites. Estos dos últimos resultados, aunque muestran niveles de cadmio por encima de lo permitido, y por tanto difieren de los hallazgos en España ${ }^{(8)}$, son inferiores a los encontrados en Noruega ${ }^{(7)}$.

Al comparar con Pérez et al. ${ }^{(5)}$ y Frías-Espericueta et al. ${ }^{(6)}$ observamos que el cadmio se está incrementando, principalmente por actividad antropogénica, y se almacena en moluscos comestibles los cuales son consumidos por los pobladores en su dieta.
Para contrastar moluscos cefalópodos se analizó pota (Dosidicus gigas) cuyo promedio fue $1,11 \mathrm{mg} /$ $\mathrm{kg}$ peso fresco, siendo ligeramente mayor al límite permitido de $1,00 \mathrm{mg} / \mathrm{kg}$ peso fresco y difiriendo del estudio catalán en donde no se superan los valores límites.

\section{CONCLUSIONES}

Las concentraciones de cadmio en pescados superan los límites permitidos según la Comisión de la Unión Europea en su Reglamento (CE) № 1881/2006 y modificatoria Reglamento (UE) $\mathrm{N}^{\circ}$ $488 / 2014$.

Las concentraciones de cadmio en crustáceos no superan los límites permitidos según la norma.

Las concentraciones de cadmio en moluscos bivalvos y moluscos cefalópodos superan ligeramente los límites permitidos según la norma.

\section{REFERENCIAS BIBLIOGRÁFICAS}

1. Gisbert C. Medicina legal y toxicología. $6^{\text {ta }}$ ed. Barcelona: Masson; 2004.

2. Valle P. Toxicología de alimentos. México: Instituto Nacional de Salud Pública; 2000.

3. Juárez H. Contaminación del río Rímac por metales pesados y efecto en la agricultura en el cono este de Lima Metropolitana. [Tesis para optar el título de Magíster en Ciencias Ambientales]. Universidad Nacional Agraria La Molina. La Molina, 2006.

4. Ibárcena W. Estudio de la contaminación por metales pesados en las costas de Tacna - Perú. Ciencia \& Desarrollo, UNJBG. 2009; (11): 113-8.

5. Pérez A, Fajardo M, Strobl A, Pérez L, Piñeiro A, López C. Contenido de plomo, cromo y cadmio en moluscos comestibles del Golfo San Jorge (Argentina). Acta Toxicol Argent. 2005; 13(1): 20-5.

6. Frías-Espericueta M, Osuna-López J, Izaguirre-Fierro G, Aguilar-Juárez M, Voltolina D. Cadmio y plomo en organismos de importancia comercial de zona costera de Sinaloa, México: 20 años de estudios. CICIMAR Oceánides. 2010; 25 (2): 27-39.

7. Nasjona ltinstitutt for ernærings- ogsjømatforskning (NIFES). Kadmium- og blyanalyser av kamskjell og oskjell. Program for overvåkning. 2008

8. Agencia Catalana de Seguridad Alimentaria. Contaminantes químicos en pescado y marisco consumido en Cataluña. Barcelona: Agencia Catalana de Seguridad Alimentaria; 2008.

9. Mauri A, Llobat M \& Herráez R. Laboratorio de análisis instrumental. Valencia: Reverte; 2010.

10. Unión Europea. Reglamento (CE) № 1881/2006 de la Comisión, de 19 de diciembre de 2006, por el que se fija 
el contenido máximo de determinados contaminantes en los productos alimenticios. Diario Oficial de la Unión Europea L364, 20 de diciembre de 2006, pp. 5.

11. Unión Europea. Reglamento (UE) № 420/2011 de la Comisión, de 29 de abril 2011, que modifica el Reglamento (CE) № 1881/2006, por el que se fija el contenido máximo de determinados contaminantes en los productos alimenticios. Diario Oficial de la Unión Europea Lı11, 30 de abril 2011, pp. 3.

12. Unión Europea. Reglamento (UE) № 488/2014 de la Comisión, de 12 de mayo de 2014, que modifica el Reglamento (CE) № 1881/2006 por lo que respecta al contenido máximo de cadmio en los productos alimenticios. Diario Oficial de la Unión Europea L138, 13 de mayo de 2014, pp. 75.
Manuscrito recibido el: 07/10/2015

Aceptado para su publicación el: 20/04/2016

\section{Correspondencia:}

Nombre: $\quad$ Gloria Marina Marín Vallejos Mesías Moisés García Ortiz

Dirección: Jr. Huancavelica $N^{\circ} 857$, Villa el Salvador. Jr. Puno $\mathrm{N}^{\circ}$ 1002, Lima.

e-mail: mmarinvallejos@yahoo.es mmoisesgarciao@gmail.com 